\title{
Modeling of Virtual Assembly and Disassembly Process Based on Selective Disassembly
}

\author{
Hao Wang ${ }^{1,2, ~ *, ~ Y a n ~ T a o ~ Y a n g ~}{ }^{2}$, Qing Shen ${ }^{1,2}$, Qian Zhang ${ }^{1,2}$, Bin Yin ${ }^{1,2}$ \\ ${ }^{1}$ National Engineering Laboratory for Marine and Ocean Engineering Power System, Shanghai, China \\ ${ }^{2}$ Power Plant Division, Shanghai Marine Diesel Engine Research Institute, Shanghai, China
}

Email address:

ee_haowong@163.com (Hao Wang)

*Corresponding author

\section{To cite this article:}

Hao Wang, Yan Tao Yang, Qing Shen, Qian Zhang, Bin Yin. Modeling of Virtual Assembly and Disassembly Process Based on Selective Disassembly. International Journal of Intelligent Information Systems. Vol. 10, No. 2, 2021, pp. 16-22. doi: 10.11648/j.ijiis.20211002.12

Received: May 2, 2021; Accepted: May 17, 2021; Published: June 3, 2021

\begin{abstract}
The virtual disassembly and assembly system based on computer virtual reality technology has been rapidly developed and applied. However, the current virtual disassembly system and assembly based on the hierarchy model and association model does not consider the problem of selective disassembly, which leads to inconsistency between virtual disassembly and actual disassembly. Aiming to solve the problem, introduces the concept of skipping disassembly path on the basis of the original disassembly system based on the hierarchical relationship and the association relationship model, and perfects and optimizes the disassembly and assembly structure model based on the hierarchical relationship model and the association relationship model. The sequence planning of the disassembly model after introducing the shipping disassembly path was carried out. And the combination and reduction of disassembling units in the course of disassembly decision was described. Finally, a selective disassembly algorithm based on the association relationship model is established. With the marine oil separator for example verification. The results show that the model can better solve the actual problem of selective disassembly which improved the authenticity and user experience of the virtual disassembly and assembly system, and has great influence on the application development of the virtual disassembly and assembly system.
\end{abstract}

Keywords: Selective Disassembly, Skipping Disassembly Path, Sequence Planning, Oil Separator

\section{Introduction}

Virtual disassembly based on Virtual Reality (VR) is the essence of the actual disassembly and assembly process on the computer. It is a process of design and analysis of equipment disassembly and assembly, planning and verification of disassembly process, training of disassembly and operation and the final realization of virtual disassembly and assembly evaluation by using computer simulation and virtual reality technology $[1,2]$. For the virtual disassembly and assembly of marine equipment, it mainly focuses on disassembly and assembly training and operation evaluation. However, the actual disassembly and disassembly needs to purchase a large number of equipment, which is expensive, and is limited by personnel (some disassembly and assembly operations need to be completed by many people, such as the disassembly and assembly of the host), site and time, and there are certain safety risks [3]. At the same time, with the development of computer software / hardware, virtual disassembly and assembly system based on virtual reality technology has become a more economical, effective and rapid alternative way. It can enable users to interact through input / output devices in virtual environment to achieve the expected training effect, greatly improve efficiency and save costs, and also make users (crew, students, et al.) familiar with the principle of equipment, accumulate the experience of disassembly and assembly, and lay the foundation for practical operation.

In order to make virtual disassembly and assembly truly reflect the essence of the actual disassembly and assembly process, the disassembly and assembly process of equipment should be modeled, including disassembly information model, coordination constraint model and disassembly structure model. At present, the modeling methods for disassembly and assembly structure model mainly include hierarchical relation 
model and association relation model and their comprehensive application, such as the equipment tree model $[4,5]$ applied in disassembly process modeling is essentially a hierarchical relationship model, and the assembly model based on attribute adjacency graph matching [6] is essentially an association relationship model. The modeling model of equipment disassembly process based on Project software [7, 8] is the comprehensive application of hierarchical relationship model and association relationship model. The above research has made a positive exploration on the process modeling method of virtual disassembly and assembly system of equipment, but it is not considered that all parts may not be removed (overall disassembly) in the actual disassembly and maintenance process, but only a specific part (selective disassembly) needs to be disassembled.

This paper introduces the concept of jumping disassembly path into the virtual disassembly and assembly structure model, improves and optimizes the existing hierarchical relation model and correlation model, solves the problem of selective disassembly in the process of disassembly and assembly, and takes the disassembly process of marine oil separator as an example to verify and analyze.

\section{Disassembly Structure Model Based on Hierarchy and Association}

In order to ensure the authenticity and effectiveness of the virtual disassembly process of equipment, it is necessary to model the disassembly process of equipment in addition to the realistic three-dimensional model. Disassembly and assembly process modeling is one of the key technologies of virtual disassembly and assembly research. The establishment of effective disassembly and assembly process model is the basis of realizing effective disassembly and assembly and optimizing disassembly and assembly process. In the process of design for disassembly (DFD) and design for assembly (DFA ) [9], the common structure models of equipment disassembly mainly include hierarchical relationship model and association relationship model. The main modeling methods include IDEF3 method [10], Petri net method [11], CPM / PERT method [12], Gantt chart method [7], Directed acyclic graph method [13], etc.

\subsection{Hierarchical Relationship Model}

In the hierarchical relationship model, the model is described according to the hierarchical relationship of "equipment $\rightarrow$ disassembly assembly $\rightarrow$ sub disassembly assembly $\rightarrow$ basic disassembly unit". An equipment can be divided into sub-assemblies and parts at different levels, as shown in "Figure 1". In the assembly process, the assembly body (component or assembly) is composed of parts first, and then participate in the assembly of the whole machine; in the disassembly process, the equipment is first decomposed into assembly body and sub assembly body, until it is decomposed to the target parts. The relationship between the components of the equipment can be represented by hierarchy. However, the description of the assembly relationship among the assembly units at the same level is not intuitive enough in the hierarchical relationship model, which cannot fully describe the constraint relationship and disassembly sequence of the actual equipment.

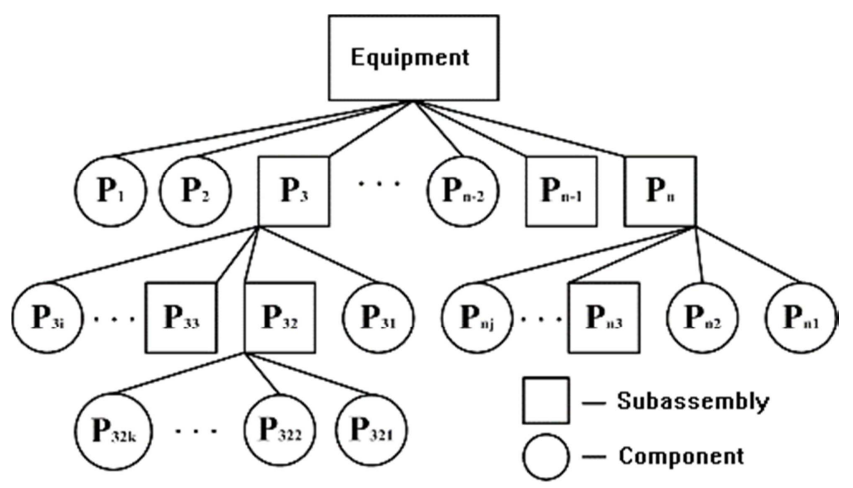

Figure 1. Hierarchical relationship model.

\subsection{Association Relationship Model}

In the association relationship model "Figure 2", nodes represent components, and the lines between nodes represent the coordination constraint relationship between components [7]. The model can be divided into directed graph and undirected graph according to the directivity of the line. In directed graph, the line between nodes is a directed edge, and the direction of the line indicates the sequence of constraints; in undirected graph, the line between nodes is an undirected edge, which cannot describe the sequence of constraints. The relational model is more intuitive, which is convenient for access management and subsequent direct use of all kinds of information. Information description is often carried out at the same level, which can well describe the assembly relationship of components at the same level, but it does not conform to the actual construction habits of the equipment, and when the number of components is large, the number of nodes increases, so it is difficult to search the algorithm.

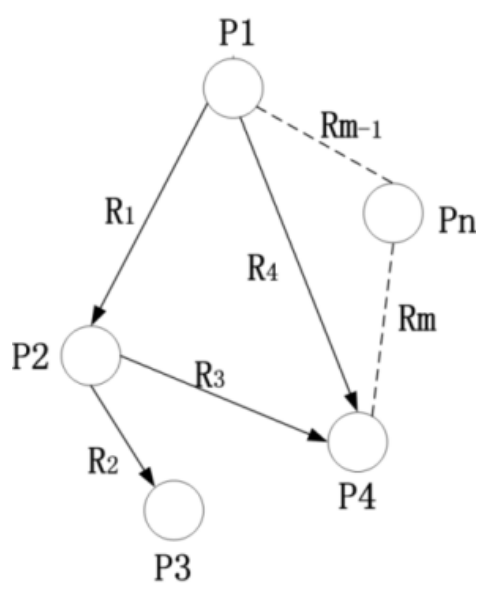

Figure 2. Association relationship model.

The relationship between assembly parts can be expressed by formula (1): 


$$
\mathrm{G}=\{\mathrm{P}, \mathrm{R}\}
$$

Where $\mathrm{P}=\{\mathrm{P} 1, \mathrm{P} 2, \ldots, \mathrm{Pn}\}$ is the set of components, and $\mathrm{R}=$ $\{\mathrm{R} 1, \mathrm{R} 2, \ldots, \mathrm{Rn}\}$ is the set of assembly relationships among components.

\section{Modeling of Selective Disassembly and Assembly Based on Hierarchical and Association Relationship}

From the above analysis, we can see that the two models have their own advantages and disadvantages, so the disassembly structure modeling often adopts the strategy of combining the association relationship model and the hierarchical relationship model. In addition, considering the characteristics of actual disassembly activities, the existing hierarchical relationship model and association relationship model are improved. Based on the hierarchical relationship and association relationship, the concept of jumping disassembly path is introduced to form a disassembly structure model to meet the selective disassembly (as shown in "Figure 3"). The matching constraint relationship between components at the same level of the model is represented by an association relationship model, which is based on the directed graph, and introduces the concepts of virtual constraint and undirected constraint as the improvement and supplement of the model to meet the needs of the actual disassembly sequence planning.

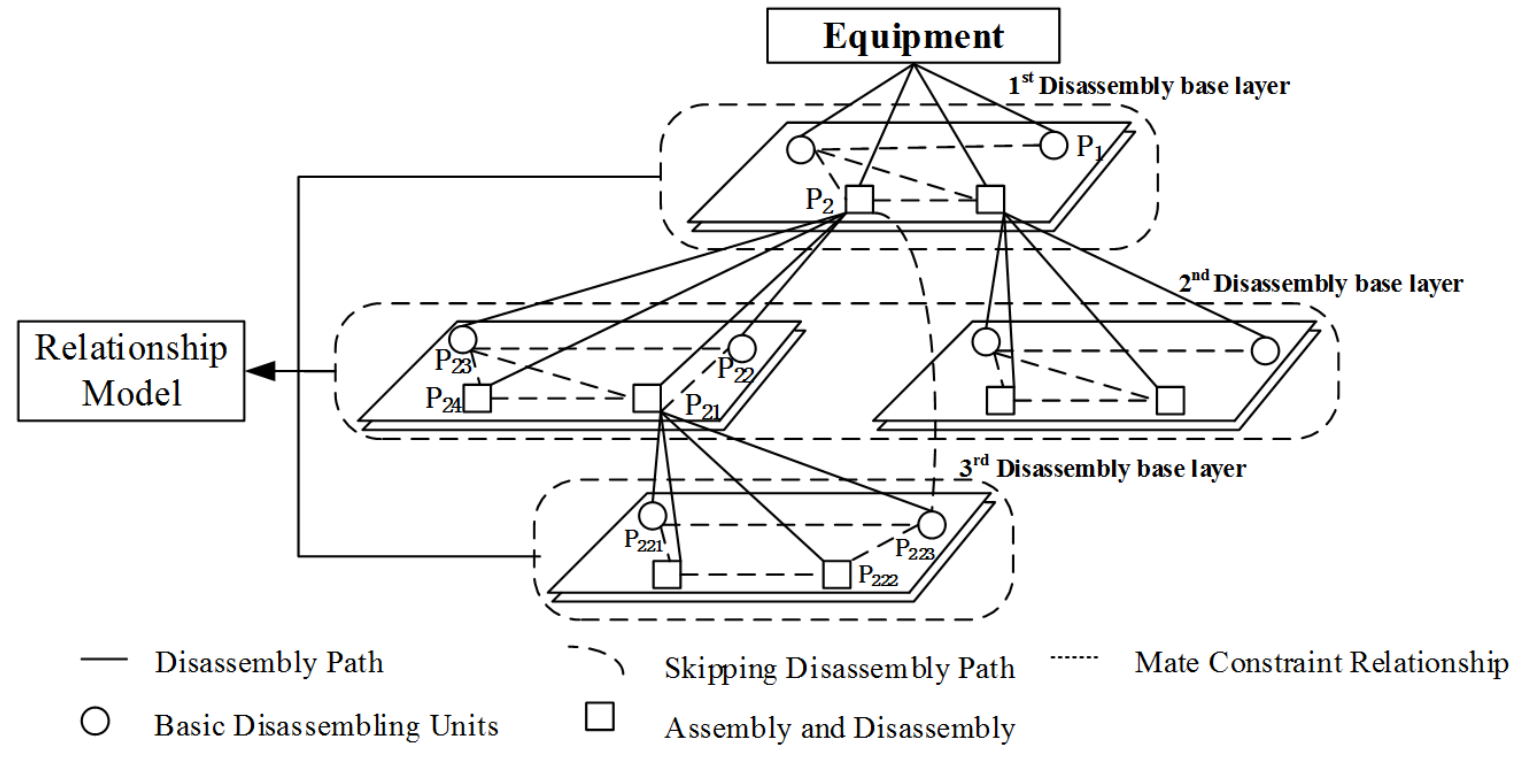

Figure 3. Selective disassembly structure model.

It can be seen from the model that in the process of disassembly and assembly of equipment, the related components are often divided into a group. When disassembling, the components or parts are disassembled as a whole, and then the components are disassembled. In this way, this group of components can be combined as a new component, ignoring the internal constraints and describing only the external constraints. As shown in "Figure 3", the disassembly units P221, P222, ... Are combined to form the sub disassembly assembly P21; the disassembly units P21, $\mathrm{P} 22, \ldots$ Are combined to form the disassembly assembly P2. When combining, the external constraints are concentrated on a new node after combination, while the internal constraints are automatically released. In the association relationship model corresponding to the disassembly level of P223, P221, P222 and P223 are combined into a new node P21, ignoring the internal constraints $\mathrm{C}$ and $\mathrm{E}$ (see "Figure 4"), and the external constraints A, B and D (see "Figure 5") are concentrated on P21 in the association relationship model corresponding to the disassembly level of P21. This can greatly simplify the disassembly model.

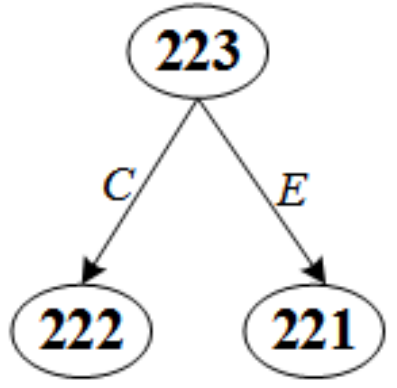

Figure 4. The association relationship model corresponding to the level of P223.

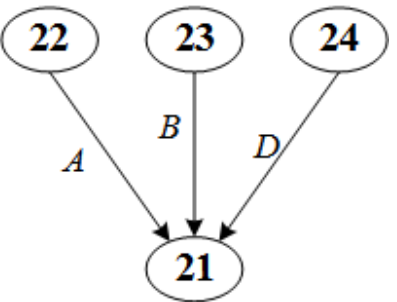

Figure 5. The association relationship model corresponding to the level of P21. 
Disassembly component and basic disassembly unit are two basic elements to describe equipment. For each disassembly component, its description structure includes two kinds of data, namely composition data and relationship data between components. The subordinate relationship of the assembly and disassembly components at all levels forms a hierarchical model, and the coordination constraint relationship between the components at the same level forms an association relationship model.

Introduction of Jumping Disassembly Path

Jumping disassembly path means that when a component can be disassembled directly from a higher level, it can be disassembled without strictly following the disassembly path determined by the hierarchy relationship. As shown in "Figure 3", when following the hierarchical structure, for disassembly unit $\mathrm{P} 21$, the disassembly path is $\mathrm{P} 2 \rightarrow \mathrm{P} 21$; for disassembly unit P223, the disassembly path is $\mathrm{P} 2 \rightarrow \mathrm{P} 21 \rightarrow \mathrm{P} 223$. However, for the selective disassembly, that is, when disassembling a certain component, consider the requirement of the principle of minimum disassembly in the disassembly process, that is, keep the parts that do not need to be disassembled as much as possible to reduce the workload of disassembly, so as to reduce the workload of disassembly. Similarly, for disassembly unit P223, the jumping disassembly path is P2-P223.

The jump disassembly path is proposed for selective disassembly. For the overall disassembly of the equipment, the jump disassembly path is not considered, and the disassembly path in the hierarchical model is still used for disassembly.

Disassembly Unit Restoration During Disassembly Decision Process

However, in the process of selective disassembly, when the disassembly path jumps (as shown by the virtual arc in "Figure 3 "), the problem of disassembly unit restoration is involved, and the restoration can be regarded as the reverse process of combination. That is to say, for the skip disassembly path P2 $\rightarrow$ P223 of disassembly unit P223, the selective disassembly of $\mathrm{P} 2$ is still carried out by using the association relationship model corresponding to the disassembly level of $\mathrm{P} 2$. At this time, P21 is restored to P221, P222 and P223, and the internal constraints $\mathrm{C}$ and $\mathrm{E}$ are restored, and the external constraints are assigned to P221, P222 and P223 accordingly. Among them, constraints $\mathrm{A}$ and $\mathrm{B}$ point to $\mathrm{P} 222$, and constraint D points to P223 (see "Figure 6"). Then the selective disassembly of P223 is carried out by using the association relationship model corresponding to the disassembly level of P223 (see "Figure 4").

It can be seen that after the disassembly unit is restored, the corresponding hierarchical relationship model and association relationship model are changed. The disassembly level of the unit is changed from three layers to two layers, and the association relationship model of the restored P21 layer is also changed from "Figure 5" to "Figure 6". Among them, the corresponding association relationship model of each level is shown in "Figure 4", "Figure 5" and "Figure 6".

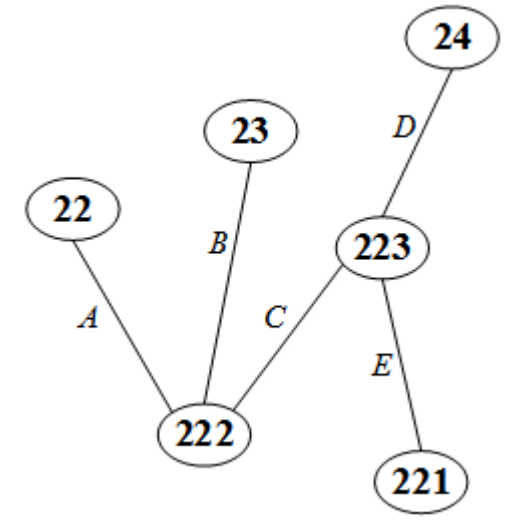

Figure 6. The association relationship model of the restored P21 level.

Selective disassembly Sequence Planning

In the target disassembly, the workload should be reduced as much as possible, that is, the unnecessary parts should be retained as much as possible, and the selective disassembly does not strictly follow the disassembly sequence of "assembly $\rightarrow$ component $\rightarrow$ component $\rightarrow$ part" implied in the hierarchical model. For selective disassembly planning, the purpose is to get the disassembly constraints and the order that must be removed in order to obtain the target parts, not the order of the parts that need to be disassembled. In the actual disassembly process, there is such a situation that when a disassembly unit is disassembled, its parent node does not need to be disassembled first, and even its parent node does not need to be disassembled. Only part of the constraints need to be removed, it can be directly disassembled from the upper or higher level of the disassembly level. Of course, if there is no jump path for the target part, the disassembly path is searched from the tree model. The disassembly sequence planning of the selective disassembly is shown in "Figure 7".

When disassembling the target component, first judge whether its parent node is the root node. If it is, call the selective disassembly algorithm to plan the disassembly of the target component according to the association relationship model corresponding to the level-1 disassembly benchmark layer in the hierarchical relationship model; if not, search the disassembly path according to the hierarchical relationship model and judge whether the nodes on the disassembly path have jump disassembly If there is a disassembly path, the disassembly path of the selective disassembly part is given after the existence of the leaping disassembly path, and the intermediate node is restored to get the restored disassembly structure model. At this time, according to the order of the nodes in the searched disassembly path, the selective disassembly algorithm is called according to the corresponding association relationship model of each node's level, and the corresponding disassembly orders of each node are respectively processed If not, according to the order of each node in the hierarchical relationship model path, and the corresponding association model of each node in the hierarchy, the selective disassembly algorithm is called to carry out the selective disassembly planning for the corresponding disassembly units. 


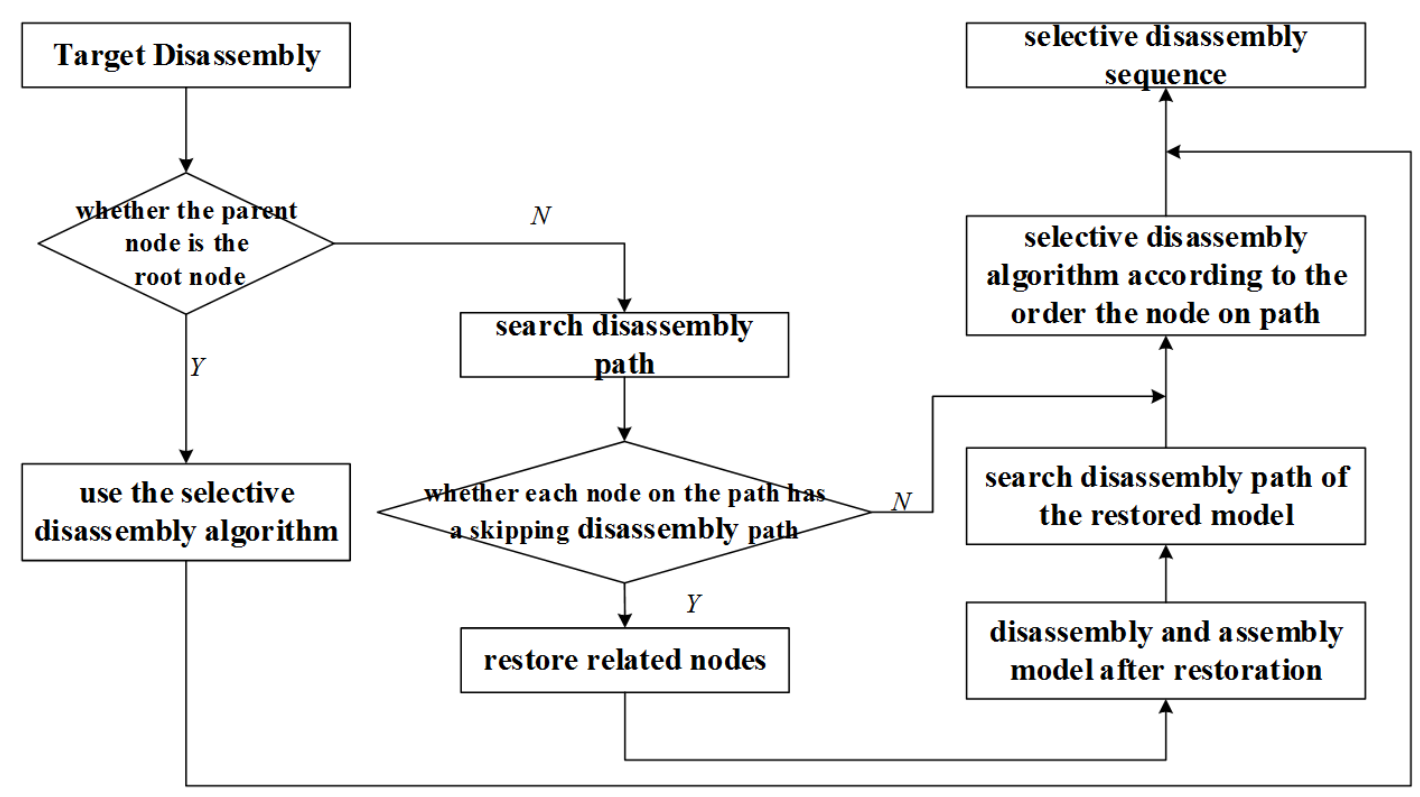

Figure 7. Selective disassembly sequence planning process.

Selective disassembly Algorithm Based on Association Relation Model

In the process of equipment disassembly sequence generation, according to the different purpose of disassembly, it can be divided into two different forms: complete disassembly sequence algorithm and selective disassembly sequence algorithm. Among them, the generation of complete disassembly sequence can be divided into breadth based disassembly sequence, depth based disassembly sequence and disassembly sequence algorithm combining the two [14]. The selective disassembly sequence of equipment can be regarded as a subsequence of complete disassembly sequence, but it is not equal to complete disassembly sequence. The generation algorithm of selective disassembly sequence generally does not use the generation algorithm of complete disassembly sequence, Because of its blindness, which will seriously reduce the work efficiency. In this paper, the disassembly sequence of target unit is generated by backstepping method. The specific steps are as follows:

Step 1. find the row corresponding to the target cell in the associated matrix $S$ of the device, and search for the unit represented by the column corresponding to the value of 1 , and store it in an array;

Step 2. Select an element in the array generated in step 1, find the row of the cell represented by the element in s, search in the row, find the cell represented by the column corresponding to the element value of 1 , and store it in a new array with the storage cell in the previous array;

Step 3. Repeat step 2 to save the found cells and the different cells stored in the previous steps to a new array;

Step 4. Cycle according to steps 1 3 until all the units searched are detachable units. At this time, the reverse sequence of the generated sequence is the corresponding disassembly sequence of the target unit. From the incidence matrix of the equipment, we can see the constraints that need to be removed to dismantle the unit. If all the elements in the row corresponding to a unit in the incidence matrix are 0 , it indicates that the unit has been disassembled; if there is no element with value of 1 in the corresponding row, it indicates that the unit corresponding to the row has only direct connection with other units in the product, that is, the unit can be disassembled; on the contrary, if there is an element with value of 1 , it indicates that the disassembly of the unit is affected by other units. The unit is not detachable.

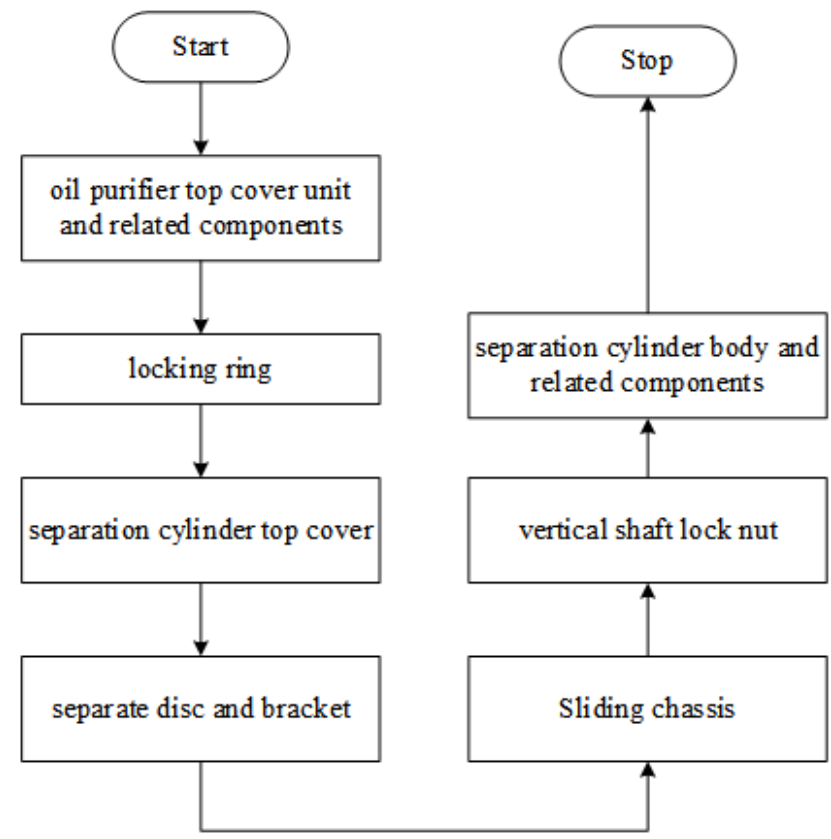

Figure 8. Disassembly and assembly flow chart of separator cylinder.

\section{Case Verification Analysis}

Oil separator is one of the purification equipment of ship 
fuel oil and lubricating oil. The operation of oil separator directly affects the safe navigation and economic benefits of the ship. Due to the frequent separation of fuel oil and lubricating oil, the disassembly, measurement, inspection and cleaning of oil separator is a regular work in the management project.

As the whole disassembly of marine oil separator is quite complex, this paper takes the disassembly of separator barrel as an example to establish its virtual disassembly process model. The whole disassembly process is shown in "Figure 8". When the selective disassembly part is encountered, the selective disassembly algorithm is called according to the flow planning diagram shown in "Figure 7".
"Figure 9" shows the disassembly process model of the separation cylinder components of the marine oil separator based on Virtools 4.0 [15], which expresses the sequence planning of the overall disassembly of the equipment to a certain extent. The disassembly sequence is basically planned according to the process shown in Figure 8. For the user specified target component, based on the whole disassembly sequence, it determines whether its parent node is the root node and whether to call the selective disassembly algorithm. For the target parts without jumping disassembly path, the disassembly is still carried out according to the overall disassembly sequence based on the hierarchical model.

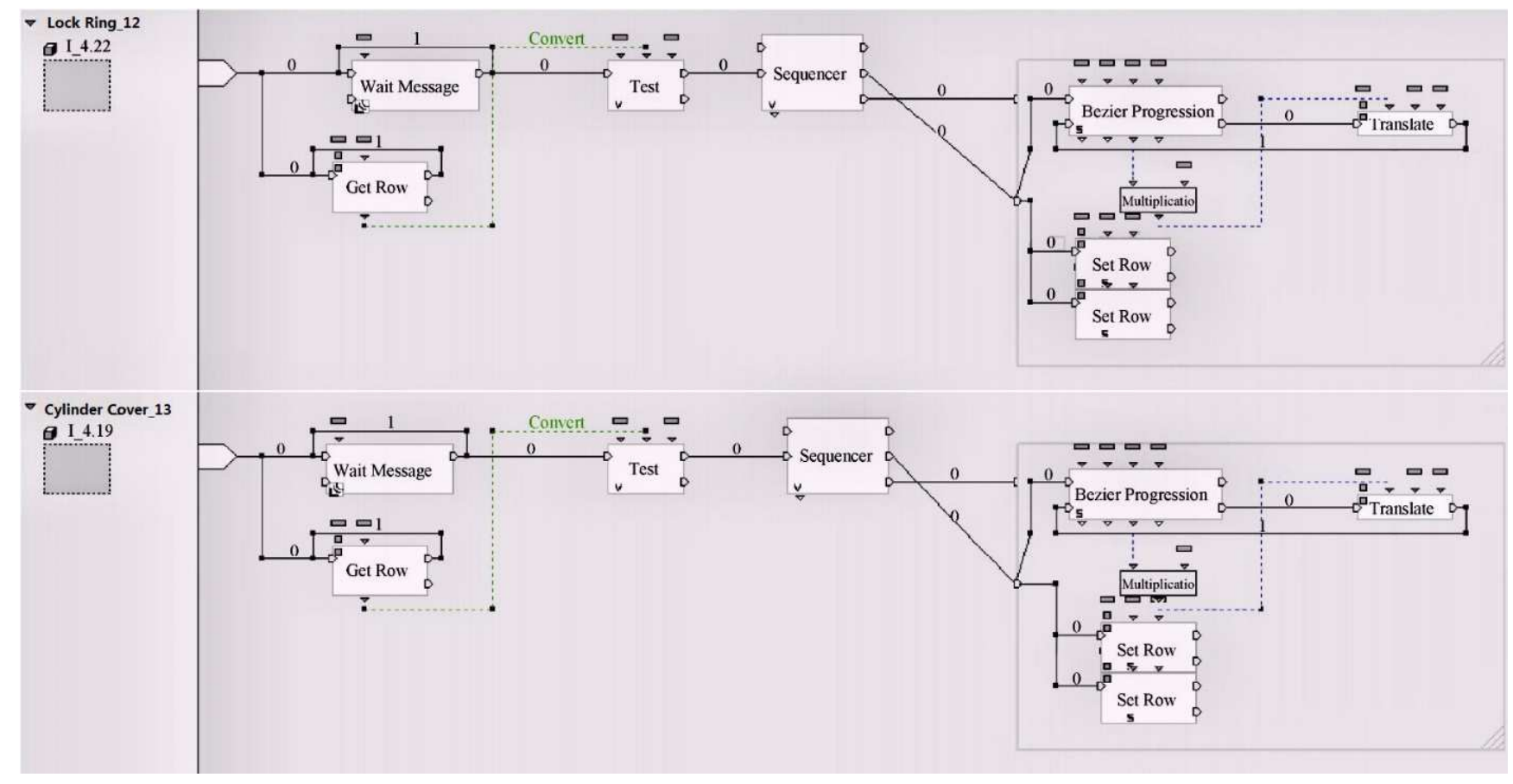

Figure 9. Disassembly process model of separation cylinder components.

Using this model can adapt to different user needs, that is, directly call the overall disassembly sequence planning process for disassembly when the overall disassembly is required; for users who require to specify the target parts, disassembly is carried out according to the model after the introduction of jump disassembly path, so as to improve work efficiency and save work time. "Figure 10" shows the actual disassembly effect of marine oil separator.

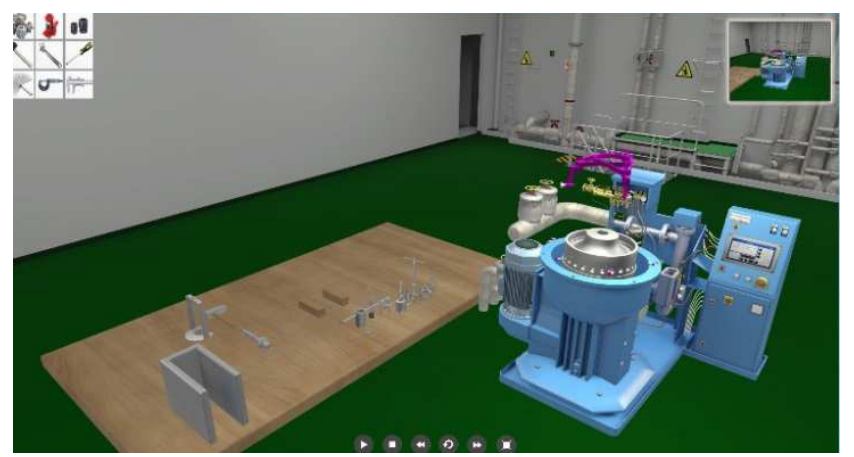

Figure 10. Virtual disassembly rendering of marine oil separator.

\section{Conclusion}

Based on the original hierarchical relationship model and association relationship model, this paper introduces the concept of jumping disassembly path to solve the problem that the selective disassembly is not considered in the disassembly structure model based on hierarchical relationship and association relationship, which makes the virtual disassembly closer to the essence of the actual disassembly process. In this paper, the disassembly structure model with jumping disassembly path is planned and the disassembly unit is restored, and the selective disassembly algorithm based on association relation model is established. Through the example modeling and verification analysis of the virtual disassembly of marine oil separator, it shows that the model can reduce the workload in the process of virtual disassembly, improve work efficiency, safety and environmental protection, and has a certain reference value for the development of related application systems.

It is hoped that experts and scholars can continue to study 
the multi-person collaborative disassembly based on this research, strive to improve the behavior path of basic disassembling units in the model and increase the calculation rate of the model to enhance user experience.

\section{References}

[1] T-W Yao, Z-R Hu \& B-W Liu. (2021). Virtual Reality Aided Maintenance Training Based on Real-time Assembly State Perception and Intuitive Interaction. [J] Chinese Journal of Engineering Design. 28 (1): 14-24. (In Chinese)

[2] W Liu. (2021). Development of Virtual Disassembly and Assembly Platform for Marine Air Compressor[J]. Journal of Physics: Conference Series. 1884 (1).

[3] H-J Geng, B-W Wang, D-L Wang, X-B Jiang. (2021). Simulated Training System of Ship Anchoring Operation Based on Virtual Reality Technology [J]. International Journal of Transportation Engineering and Technology. 7 (1): 12-18.

[4] H Zeng, X-H Wang \& J-D Zhang. (2014). Development of Virtual Disassembly and Assembly Examination System of Marine Engine for Practical Operation Evaluation [J]. China Navigation, 37 (1): 24-28. (In Chinese)

[5] Z-L Zhong, W-W Wu \& Y Zhao. (2010). Product Assembly Model for Virtual Disassembly and Assembly and Its Application [J]. Manufacturing Automation, 32 (7): 176-178, 232. (In Chinese)

[6] S-Q Tao, Z-D Huang. (2011). Assembly Model Search Method Based on Attribute Adjacency Graph Matching [J]. Chinese Journal of Computer Aided Design and Graphics, 23 (2): 290-297, 304. (In Chinese)

[7] T Huang, Y-N Zhang \& H-F Li. (2012). Modeling of Virtual Maintenance Disassembly Process Based on Project [J]. Journal of System Simulation, 24 (1): 192-196. (In Chinese)

[8] Shijie Liu, Huimin Sun, Jilin Zhang, Baifeng Liu, Zili Wang.
Study on Virtual Assembly and Disassembly Technology in Practical Teaching of Fuel Injector of Marine Auxiliary Boiler [A]. Advanced Science and Industry Research Center. Proceedings of 2019 2nd International Conference on Mathematics, Modeling and Simulation Technologies and Applications (MMSTA 2019) [C]. Advanced Science and Industry Research Center: Science and Engineering Research Center, 2019: 4.

[9] Arisya Khalda Fadhilah, Suryantini Rini. (2021). Modularity in Design for Disassembly (DfD): Exploring the Strategy for a Better Sustainable Architecture [J]. IOP Conference Series: Earth and Environmental Science. 738 (1).

[10] N Dong, K Ning \& Q Li. (2001). Process Modeling Method Based on IDEF3 and Its Supporting Tools [J]. Computer Integrated Manufacturing System, 7 (12): 30-34. (In Chinese)

[11] J-X Liu, T Liu \& H Geng. (2013). Modeling and Application of Virtual Maintenance Process Based on Petri Net and Semantic Network [J]. Journal of Graphics, 34 (2): 113-118. (In Chinese)

[12] J Shang, X-F Rong \& X-H Xu. (2011). Virtual Disassembly Process Modeling Based on PERT Diagram [J]. Computer and Digital Engineering, 39 (3): 151-154. (In Chinese)

[13] X-X Zheng, G-Z Sun \& S-M Wang. (2005). Researches on Virtual Prototyping Presentation Model Based on Directed Acyclic Graph [C]. The 9th International Conference on Computer Supported Cooperative Work in Design Proceedings. 633-637.

[14] Z-P Fu, H-Y Zhao \& Y-X Wang. (2017). Disassembly Sequence Generation Algorithm Based on Dynamic Search [C]. Proceedings of 2017 Academic Conference on System Simulation Technology and Its Application. 822-825. (In Chinese)

[15] Zhen Tian, H Jiang, X-D Tan, L Chen. (2018). Development of Virtual Assembly System of Truck Drive Axle Based on 3DMAX and Virtools [J]. Equipment Manufacturing Technology. (08): 142-144. (In Chinese) 